Abanico Veterinario. Enero-Diciembre 2020; 10:1-9. http://dx.doi.org/10.21929/abavet2020.12 Artículo Original. Recibido: 17/02/2020. Aceptado: 10/06/2020. Publicado: 25/06/2020. Clave: $2020-21$.

\title{
Comparación de tres sitios de muestreo sanguíneo para la evaluación fisiometabólica en el lechón
}

\section{Comparison of three blood sampling sites, for physiometabolic evaluation piglets}

\section{Sánchez-Salcedo José1 ID, González-Lozano Miguel2 ID, Bonilla-Jaime Herlinda $^{3 I D}$, González-Hernández Milagros ${ }^{4}$ ID, Ballesteros-Rodea Gilberto ${ }^{4}$, Orozco-Gregorio Héctor 4 ID}

\begin{abstract}
${ }^{1}$ Universidad Autónoma Metropolitana, Doctorado en Ciencias Biológicas y de la Salud, Ciudad de

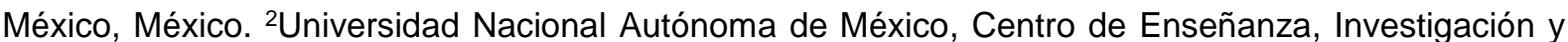
Extensión en Producción Porcina, Jilotepec, Estado de México. ${ }^{3}$ Universidad Autónoma Metropolitana, Departamento de Biología de la Reproducción, Ciudad de México, México. ${ }^{4}$ Universidad Autónoma de San Luis Potosí, Facultad de Agronomía y Veterinaria, San Luis Potosí, México. Autor responsable: Sánchez-Salcedo José. Autor de correspondencia: Orozco-Gregorio Héctor. Centro de Enseñanza, Investigación y Extensión en Producción Porcina, Universidad Nacional Autónoma de México. Km. 2 Carretera Jilotepec-Corrales S/N, Col. La Dalia, 54240. Jilotepec, Estado de México, México. jsanchezsalcedo@xanum.uam.mx, migueglozano@yahoo.com.mx, bjh@xanum.uam.mx, milagros.gonzalez@uaslp.mx, gilberto.ballesteros@uaslp.mx, hector.orozco@uaslp.mx
\end{abstract}

\section{RESUMEN}

La extracción sanguínea en los lechones neonatos es necesaria para la evaluación fisiometabólica y para determinar alteraciones gaseosas útiles en el diagnóstico de varios fenómenos como la asfixia intraparto; siendo la causa principal no infecciosa que origina una alta tasa de mortalidad neonatal en cerdos. Nuestro objetivo fue evaluar tres de los sitios más comunes de muestreo sanguíneo usados en lechones: cordón umbilical (UC), seno retro orbital (ROS) y vena cava (VC); así como determinar las diferencias entre ellos con respecto a las variables fisiometabólicas y el equilibrio ácido-base. Treinta y dos lechones sanos provenientes de nueve partos eutócicos fueron aleatoriamente seleccionados para los tres sitios de muestreo. Todas las concentraciones fisiometabólicas mostraron valores basales de los parámetros fisiológicos fetales correspondientes al sitio de muestreo. Sin embargo, los valores de UC resultaron más confiables para el diagnóstico del intercambio gaseoso durante la asfixia, debido a su similitud con los parámetros fisiológicos de un neonato sano $\left(\mathrm{pCO}_{2}=36.10 \pm 2.03, \mathrm{pO}_{2}=32.68 \pm\right.$ 3.03); mientras que para el perfil metabólico (glucosa y lactato) los valores de ROS fueron más precisos en determinar la glicemia y el lactato, comparados con los demás sitios. Por consiguiente, cuando el perfil fisiometabólico neonatal es requerido, el abordaje metodológico en el sitio de muestreo tiene que ser específico, de acuerdo a los objetivos planteados y a su subsecuente interpretación.

Palabras clave: lechones, gases sanguíneos, cordón umbilical, seno retroorbital y vena cava.

\section{ABSTRACT}

Blood drawing of newborn piglets is necessary for physio-metabolic profiles assessment, and to determine gas change levels in it, what is used in various phenomena diagnosis, such as, intrapartum asphyxia a non-infectious condition that causes a high rate of neonatal mortality in pigs. This study aimed to test three of the most common blood sampling sites used in piglets: umbilical cord (UC), retroorbital sinus (ROS), and cava vein (VC), besides determining the differences among them, concerning to physio-metabolic variables and the acid-base balance. Thirty-two healthy non asphyxiated piglets were randomly selected, coming from nine eutocic farrowing to carry out the three sampling sites. All of the physio-metabolic concentrations determined showed basal values within normal fetal ranges corresponding to the sampling site. However, UC values were more suitable for gas exchange diagnosis in asphyxia due to their similarity with physiological parameters of a normal newborn ( $\mathrm{pCO} 2=36.10 \pm$ 
$2.03, \mathrm{pO} 2=32.68 \pm 3.03$ ), whereas for the metabolic profile (glucose and lactate) ROS values were more accurate for measuring a common lactate and glycaemia profile than the other sites. Therefore, when a physio-metabolic profile is required, the methodological approach of the blood sampling site should be adequate to obtain the data needed for research following planned objectives and subsequent interpretation.

Keywords: newborn pig, blood gasses, umbilical cord, retro orbital sinus, vena cava.

\section{INTRODUCCIÓN}

En producción porcina, existe una tasa elevada de mortalidad neonatal, la cual repercute directamente en el ámbito económico y sobre el bienestar de los animales. Al respecto, es ampliamente aceptado que de manera práctica no ha habido una disminución significativa en el número de mortinatos durante los últimos treinta años, donde se estima que la mortalidad promedio fluctúa entre 16\% y $20 \%$ (Baxter y Edwards, 2018); de los cuales el $8 \%$ de las muertes corresponden a procesos de asfixia fetal y neonatal (Vanderhaeghe et al., 2013; van Dijk et al., 2005). Por consiguiente, la asfixia intraparto es considerada como la causa principal de origen no infeccioso que subyace la muerte de un número considerable de lechones dentro de las primeras 72 horas postparto (Baxter et al., 2011).

Algunas características propias de los lechones, como la integridad del cordón umbilical, el orden de expulsión durante el parto y el peso al nacimiento, pueden predisponer a la aparición de asfixia intraparto (Sánchez-Salcedo et al., 2019b). Dichas alteraciones pueden originar cambios en la ventilación pulmonar neonatal, lo que a su vez provoca una disminución en el flujo de oxígeno al cerebro (Herrera-Marschitz et al., 2014), conduciendo a un posible daño neurológico y una adquisición deficiente de inmunidad pasiva, con la incapacidad posterior para aumentar de peso durante el periodo postnatal (Orozco-Gregorio et al., 2012). Aunado a lo anterior, los costos asociados con la mortalidad predestete, desde la gestación hasta el parto, resultan cercanos a los 45.72 dólares (Seddon et al., 2013); por lo que existe una necesidad imperiosa de desarrollar estrategias terapéuticas encaminadas a la prevención o al tratamiento de esta condición.

Es por esto, que para el diagnóstico correcto de las alteraciones respiratorias de los lechones y para una adecuada intervención terapéutica, es necesaria la evaluación del perfil fisiometabólico y de las concentraciones de gases sanguíneos, como un indicador confiable del pronóstico de viabilidad postparto de los neonatos. No obstante, la extracción sanguínea de un lechón recién nacido es difícil, debido a las condiciones inherentes del nacimiento (presencia de fluidos en el cuerpo, tamaño pequeño y movimientos rápidos); por lo que el sitio y el tiempo de muestreo deben ser precisos. Al respecto, el análisis de gases en sangre se puede realizar a través de sangre arterial o venosa, siendo la opción venosa el procedimiento más seguro y fácil en animales, como los cerdos. Sin embargo, al ser una especie politoca, se puede esperar una amplia gama de variables fisiometabólicas al momento de su nacimiento 
en diferentes miembros de la misma camada (Orozco-Gregorio et al., 2012), lo cual hace necesario un diagnóstico individualizado.

Por lo tanto, el objetivo de la presente investigación fue evaluar tres sitios diferentes de muestreo sanguíneo, para la determinación de los perfiles fisiometabólicos en lechones recién nacidos sin evidencia de asfixia y sus diferencias entre los diversos sitios de muestreo: cordón umbilical (UC), seno retro orbital (ROS) y vena cava (VC); con la finalidad de determinar su idoneidad al momento de la toma de decisiones para el diagnóstico de la asfixia intraparto.

\section{MATERIAL Y MÉTODOS}

Condiciones del estudio. Para la realización del presente trabajo se emplearon lechones sanos sin evidencia de asfixia $(n=32)$, nacidos de 9 cerdas multiparas con partos eutócicos (3.6 \pm 0.32 partos; peso: $200-280 \mathrm{~kg}$ ) de la raza híbrida YorkshireLandrace; con la finalidad de determinar las diferencias basales entre los tres sitios de muestreo. Todas las cerdas se encontraron alojadas en el Centro de Enseñanza, Investigación y Extensión en Producción Porcina de la Universidad Nacional Autónoma de México (CEIEPP-UNAM), bajo condiciones normales de maternidad (dieta estándar y agua ad libitum en jaulas individuales de maternidad con temperatura controlada de $23 \pm 2 \stackrel{\circ}{\circ}$ ).

Todos los procedimientos realizados con animales fueron aprobados por el Comité Interno para el Cuidado y Uso de los Animales de la Facultad de Medicina Veterinaria y Zootecnia (CICUA-FMVZ-UNAM), con el número de aprobación de protocolo 062. Además, durante la realización de todas las pruebas se siguieron las directrices nacionales para el uso ético de animales para experimentación.

Procedimientos experimentales. Las cerdas fueron aleatoriamente seleccionadas para el muestreo de sus lechones (UC: $n=12$ lechones, ROS: $n=10$ lechones y VC: $n=10$ lechones). Todos los partos fueron inducidos 24 horas previas a la fecha probable de parto (día 115 de gestación) con $1 \mathrm{~mL}$ intramuscular de cloprostenol (Bioestrophan, Laboratorios Syva, Querétaro, México), según las prácticas rutinarias de la unidad de producción. Al nacimiento ningún lechon recibió atención médica ni intervenciones terapéuticas, con la finalidad de no interferir en el proceso natural del parto.

Inmediatamente después del nacimiento y en un tiempo no mayor a 10 segundos, las muestras sanguíneas de UC y VC fueron tomadas mediante una punción con jeringas heparinizadas (INHEPAR $5000 \mathrm{UI} / \mathrm{mL}$, PiSA Farmaceutica, México; agujas 23 g x 1'); mientras que las muestras correspondientes a ROS fueron tomadas mediante tubos capilares de borosilicato también heparinizados (Orozco-Gregorio et al., 2008; (Sánchez-Salcedo et al., 2019 b). Inmediatamente después de la extracción sanguínea, las concentraciones de glucosa $(\mathrm{mg} / \mathrm{dL})$, lactato $(\mathrm{mg} / \mathrm{dL}), \mathrm{pH}$ y las presiones parciales de dióxido de carbono $\left[\mathrm{pCO}_{2}(\mathrm{~mm} \mathrm{Hg})\right]$ y oxígeno $\left[\mathrm{pO}_{2}(\mathrm{~mm} \mathrm{Hg})\right]$ fueron cuantificadas, usando un gasómetro clínico automatizado (Epocal Inc., Ottawa, Canada). 
Analisis estadístico. Para el análisis de los resultados, los valores fueron expresados como la media \pm EE para las concentraciones sanguíneas de $\mathrm{pCO}_{2}, \mathrm{pO}_{2}$, glucosa, lactato y $\mathrm{pH}$ en cada uno de los tres sitios de muestreo. Los datos se analizaron utilizando un análisis de varianza de una vía (ANOVA), seguida de la prueba de HolmSidak como post-hoc. Para todas las variables los valores de $P<0.05$ fueron considerados como significativos.

\section{RESULTADOS Y DISCUSIÓN}

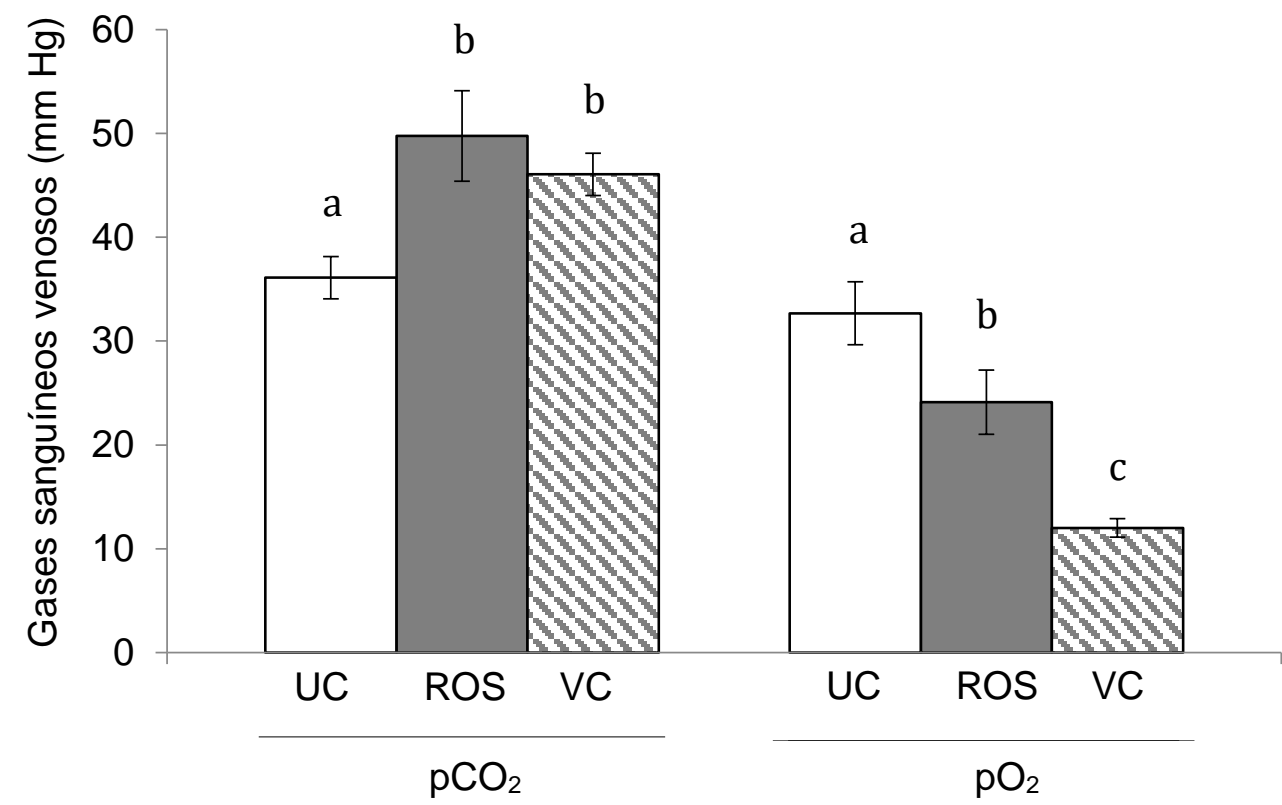

Figura 1. Valores basales del intercambio gaseoso $(\mathrm{mm} \mathrm{Hg})$ al nacimiento de tres sitios diferentes en lechones neonatos sin asfixia. Diferentes letras $a, b, c$ dentro del mismo grupo indican diferencias significativas $(P<0.05)$. Se muestran valores como medias \pm EE y prueba de Holm-Sidak como post-hoc, $(P<0.05)$

Todas las cerdas empleadas para la evaluación fisiometabólica de sus lechones tuvieron partos eutócicos, con duraciones aproximadas de $305.06 \pm 50.5$ minutos y un promedio de $11.80 \pm 0.68$ lechones vivos por camada (camadas de $12.30 \pm 0.59$ lechones en total).

Durante el proceso de parto todos los fetos experimentan periodos de hipoxia de severidad variable; los cuales, de no ser interrumpidos por el inicio de la respiración espontánea al momento del nacimiento pueden conllevar a procesos de apnea neonatal y derivar en asfixia (Sánchez-Salcedo et al., 2019ª).

Es importante remarcar que previo al parto, el pH fetal esperado es aproximadamente de 7.35, para posteriormente disminuir de manera fisiológica a valores cercanos a 7.25; siendo ambos valores considerados como normales. No obstante, los valores de $\mathrm{pH}$ entre 7.25 y 7.20 son tomados en cuenta como subnormales, por lo que requieren monitoreo; mientras que aquellos menores a 7.20 indicarán la presencia inminente de hipoxia fetal (Sánchez-Salcedo et al., 2019b).

Como se pudo evidenciar en nuestros resultados, solo aquellos valores de $\mathrm{pH}$ obtenidos a partir de UC y VC fueron concordantes con perfiles de lechones no 
asfixiados (tabla 1); mientras que las muestras tomadas de ROS arrojaron valores promedio sugerentes de asfixia $(\mathrm{pH}: 7.19 \pm 0.06)$, sin que los lechones mostraran signología propia del proceso; por lo que dicho sitio de muestreo no resulta confiable para un abordaje diagnóstico de esta alteración, ya que podemos inferir que podría ser incluso menor, bajo condiciones de asfixia en los recién nacidos. Adicionalmente, para diagnosticar un proceso como la asfixia en el cual se encuentra involucrado el intercambio gaseoso, los valores de $\mathrm{pH}$ tendrán que guardar relación directa con el oxígeno y el dióxido de carbono.

El intercambio gaseoso es un aspecto fundamental para la evaluación fisiometabólica de los lechones neonatos, debido a que dichas variables nos permiten evaluar el grado de asfixia durante el parto, al mismo tiempo que predecir la supervivencia en el periodo postparto (Orozco-Gregorio et al., 2008). Con respecto a las variables del intercambio gaseoso en el presente trabajo (figura 1), los valores de $\mathrm{pCO}_{2}$ fueron significativamente mayores en las muestras obtenidas a partir de ROS y VC (49.76 \pm 4.35 y $46.06 \pm 2.03$, respectivamente), en comparación con aquellas provenientes de UC (36.10 $\pm 2.03, P<0.05)$. Lo anterior, posiblemente se deba a que el estado ácidobase neonatal se encuentra mejor reflejado en la circulación arterial umbilical; mientras que el contenido gaseoso a partir de la sangre venosa umbilical (como se tomó en este protocolo), depende principalmente del estado ácido-base materno y de las funciones placentarias; más no directamente del neonato (Yli y Kjellmer, 2016). Por consiguiente, los valores obtenidos para $\mathrm{pCO}_{2}$ a partir de las muestras de UC no resultan de utilidad diagnóstica para los criterios aceptados para un neonato asfíctico (hipercapnia y acidosis), ya que no concuerdan con el pH del mismo sitio de muestreo, el cual corresponde a un perfil sano $(7.34 \pm 0.04)$, e incluso con valores por encima de la media. De manera contraria, los valores reportados a partir de ROS y VC para $\mathrm{pCO}_{2}$, sí pueden ser asociados con aquellos descritos por Orozco-Gregorio et al., (2012) para lechones sanos no asfixiados durante el parto y 24 horas después (60.4 \pm 18.7 and $42.1 \pm 2.2 \mathrm{~mm} \mathrm{Hg}$, respectivamente) .

En contraste, los valores de la $\mathrm{pO}_{2}$ de UC, fueron aproximadamente tres veces mayores que aquellos obtenidos a partir de VC $(32.68 \pm 3.03 \mathrm{~mm} \mathrm{Hg} \mathrm{VS} 12.01 \pm 0.89$ $\mathrm{mm} \mathrm{Hg}, P<0.001$, respectivamente). En nuestro estudio los valores de lechones sanos a partir de VC fueron más bajos que aquellos reportados para neonatos con asfixia (21.7 $\pm 10.5 \mathrm{~mm} \mathrm{Hg}$ ) por Orozco-Gregorio et al., (2012), sin un perfil asfíctico en su totalidad (valores de $\mathrm{pH}$ y $\mathrm{pCO}_{2}$ dentro de rangos normales); lo que confirma que dichos animales no fueron neonatos asfixiados; sin embargo, su $\mathrm{pO}_{2}$ fue la más baja. De manera contraria, el muestreo de ROS mostró valores $50 \%$ más altos que VC para la misma variable $\left(\mathrm{pO}_{2}: 24.12 \pm 3.09 \mathrm{~mm} \mathrm{Hg} \mathrm{VS} 12.01 \pm 0.89 \mathrm{~mm} \mathrm{Hg}, P<\right.$ $0.05)$, pero más bajos que UC (32.68 $\pm 3.03 \mathrm{~mm} \mathrm{Hg}, P<0.05)$; mientras que el oxígeno en ROS de la misma manera que en VC, exhibió concentraciones sugerentes de asfixia intraparto (<26.4 $\pm 17.7 \mathrm{~mm} \mathrm{Hg}$ ), lo que puede generar confusión, y por lo tanto conducir a un diagnóstico erróneo, ya que nuestros datos en conjunto no coinciden con los criterios de inclusión diagnóstica de un lechón con asfixia. Sin embargo, es 
ampliamente aceptado que el parto aumenta el riesgo de presentar una oxigenación comprometida por parte del feto, lo que puede conducir a interrupciones en el intercambio gaseoso, donde ciertos grados de hipoxemia y acidemia son normales; incluso en fetos sanos durante un parto eutócico (Yli y Kjellmer, 2016).

En nuestros hallazgos para la $\mathrm{pO}_{2}$, las diferencias entre los tres sitios de muestreo corresponden más a una condición fisiológica que a una patológica. En la sangre de UC, la concentración más alta de oxígeno proporcionada al feto está a una presión de sólo $30-35 \mathrm{~mm} \mathrm{Hg}$. Posteriormente, el $50 \%$ de la sangre venosa proveniente de UC pasa a través de la circulación hepática y se mezcla con sangre poco oxigenada, para pasar desde la vena cava anterior a la circulación pulmonar; por lo que la muestra obtenida de VC (12-24 mm Hg) será normalmente menos oxigenada (Hall, 2016). De manera inversa, la parte superior del cuerpo en los fetos (ROS), es exclusivamente irrigada con sangre proveniente del ventrículo izquierdo que tiene una $\mathrm{pO}_{2}$ ligeramente más alta que la sangre perfundida hacia la parte inferior del feto (Hall, 2016).

Cuando en cualquier tejido hay concentraciones de oxígeno subóptimas, hay una pérdida de la capacidad de fosforilación oxidativa y una transición resultante de un estado metabólico aeróbico a uno anaeróbico. En condiciones anaeróbicas, el piruvato es reducido a lactato, lo que lleva a una transferencia de energía ineficiente en el neonato (Yli y Kjellmer, 2016).

Nuestras concentraciones de lactato obtenidas en ROS fueron significativamente mayores $(60.66 \pm 8.51 \mathrm{mg} / \mathrm{dL})$, que aquellas de UC y VC $(41.36 \pm 5.08 \mathrm{mg} / \mathrm{dL}$ and $35.21 \pm 7.51 \mathrm{mg} / \mathrm{dL}, P<0.01$, respectivamente). No obstante, nuestros valores de ROS son consistentes con aquellos reportados por Orozco-Gregorio et al., (2008) para neonatos sanos $(65.3 \pm 15.5 \mathrm{mg} / \mathrm{dL})$, que también fueron colectados de ROS. Al respecto, es sabido que el lactato también puede funcionar como un sustrato energético cerebral en un feto sano bajo condiciones hipóxicas transitorias e inherentes a un trabajo de parto típico, consumiendo incluso más sustrato que un metabolismo oxidativo (Boardman y Hawdon, 2015).

Tabla 1. Variables sanguíneas al parto tomadas de tres sitios diferentes de muestreo en lechones neonatos sin evidencia de asfixia

\begin{tabular}{cccc}
\hline Variable & UC & ROS & VC \\
\hline $\mathrm{pH}$ & $7.34 \pm 0.04 \mathrm{a}$ & $7.19 \pm 0.06 \mathrm{a}$ & $7.27 \pm 0.01 \mathrm{a}$ \\
Glucosa $(\mathrm{mg} / \mathrm{dL})$ & $38.91 \pm 2.63 \mathrm{a}$ & $43.60 \pm 5.37 \mathrm{a}$ & $38.20 \pm 3.89 \mathrm{a}$ \\
& $41.36 \pm 5.08 \mathrm{a}$ & $60.66 \pm 8.51 \mathrm{~b}$ & $35.21 \pm 7.51 \mathrm{a}$
\end{tabular}

Medias $\pm E E . \quad U C=$ cordón umbilical $\mathrm{ROS}=$ seno retro orbital $\mathrm{VC}=$ vena cava. ${ }^{\mathrm{a}, \mathrm{b}}$ Diferentes letras en la misma fila indican diferencias significativas entre el sitio de muestreo $(P<0.001)$ 
La glucosa es considerada como la mayor fuente de energía para los lechones neonatos, debido a que dichos animales nacen con un almacenamiento energético limitado; de tal forma que, si la suplementación de glucosa y los requerimientos de ésta se encuentran equilibrados, los fetos obtendrán una oxigenación adecuada para metabolizar la glucosa aeróbicamente y transferir la energía requerida para las funciones de los órganos (Yli y Kjellmer, 2016). Sin embargo, las concentraciones de glucosa en sangre reportadas por diferentes estudios no son comparables con nuestros hallazgos, ya que son considerablemente mayores [UC: $52.25 \pm 0.12 \mathrm{mg} / \mathrm{dL}$ (Rootwelt et al., 2014); ROS: $89.3 \pm 11.4 \mathrm{mg} / \mathrm{dL}$ (Orozco-Gregorio et al., 2008); VC: $62.3 \pm 8.9 \mathrm{mg} / \mathrm{dL}$ (Trujillo-Ortega et al., 2007)], en comparación con nuestros resultados (tabla 1). Al respecto, es necesario tener en cuenta que nuestras muestras sanguíneas se obtuvieron inmediatamente después del nacimiento, con los lechones todavía en apnea (<10 segundos postparto).

Es ampliamente aceptado que una caída significativa en la glucosa durante el parto demuestra la rapidez con que se agotan las reservas de este sustrato energético. En este punto, el feto (y luego el neonato) necesita mantener la normoglucemia a través de la glucólisis anaeróbica, debido a su estado hipóxico (Martz et al., 2017); por lo que el cerebro neonatal inmaduro en comparación con un cerebro adulto maduro, es relativamente más resistente a la lesiones causadas por la hipoglucemia (glucosa < $40 \mathrm{mg} / \mathrm{dL}$ ), haciendo frente a dicha alteración mediante la disminución de los requerimientos energéticos cerebrales, aumentando el flujo sanguíneo a nivel encefálico y la movilización de glucosa; mejorando por consiguiente la capacidad de utilizar al lactato como fuente de energía alternativa (Basu et al., 2009).

Los resultados del presente informe indican que entre los tres sitios de muestreo sanguíneo para la evaluación fisiometabólica de los lechones neonatos, la diferencia principal radica en la dinámica de los gases en sangre, debido a un proceso fisiológico normal. Otro aspecto considerable es el enfoque metodológico empleado, donde la toma de muestras sanguíneas a partir de CU resulta ser el único procedimiento no invasivo en cerdos recién nacidos, con la limitación de poder obtener sangre mezclada (arterial y venosa), en comparación con ROS y VC, donde ambos pueden confirmar una muestra venosa, pero no el bienestar de los animales, si el muestreo es realizado por personal no capacitado. De la misma forma, tanto ROS como VC pueden proporcionar valores significativamente elevados y disminuidos respectivamente en caso de requerir un diagnóstico metabólico del neonato, basado en glucosa y lactato, por lo que su uso deberá de determinarse según las necesidades de la investigación, ya que no serían del todo funcionales para evaluar las capacidades respiratorias de los recién nacidos. No obstante, e independientemente del sitio de muestreo, el diagnóstico correcto de la asfixia intraparto requiere necesariamente el cumplimiento de los criterios de hipercapnia, acidosis e hipoxia, para ser considerado como una herramienta útil en producción. 


\section{CONCLUSIÓN}

Con la finalidad de determinar el sitio idóneo de muestreo sanguíneo para el diagnóstico de la asfixia intraparto, se puede concluir que los valores del cordón umbilical resultaron más confiables para el diagnóstico del intercambio gaseoso durante la asfixia, debido a su similitud con los parámetros fisiológicos de un neonato sano $(\mathrm{pCO} 2=36.10 \pm 2.03, \mathrm{pO} 2=32.68 \pm 3.03)$; mientras que para el perfil metabólico los valores de seno retro orbital fueron más precisos en determinar la glicemia y el lactato.

\section{LITERATURA CITADA}

BASU P, Som S, Choudhuri N, Das H. 2009. Contribution of the blood glucose level in perinatal asphyxia. European Journal of Pediatrics. 168:833-838. ISSN: 1432-1076. https://doi.org/10.1007/s00431-008-0844-5

BAXTER EM, Edwards AS. 2018. Piglet mortality and morbidity. En: Advances in Pig Welfare. Elsevier. Pp. 73-100. ISBN: 9780081011195. https://doi.org/10.1016/B9780-08-101012-9.00003-4

BAXTER EM, Jarvis S, Sherwood L, Farish M, Roehe R, Lawrence AB, Edwards SA. 2011. Genetic and environmental effects on piglet survival and maternal behaviour of the farrowing sow. Applied Animal Behaviour Science. 130:28-41. ISSN: 0168-1591. https://doi.org/10.1016/j.applanim.2010.11.020

BOARDMAN JP, Hawdon JM. 2015. Hypoglycaemia and hypoxic-ischaemic encephalopathy. Developmental Medicine \& Child Neurology. 57:29-33. ISSN: 14698749. https://doi.org/10.1111/dmcn.12729

HALL JE. 2016. Guyton y Hall. Compendio de Fisiología Médica. Elsevier Health Sciences Spain. Pp. 157-166. ISBN: 9788491139253.

HERRERA-MARSCHITZ M, Neira-Pena T, Rojas-Mancilla E, Espina-Marchant $P$, Esmar D, Perez R, Muñoz V, Gutierrez-Hernandez M, Rivera B, Simola N, Bustamante D, Morales P, Gebicke-Haerter PJ. 2014. Perinatal asphyxia: CNS development and deficits with delayed onset. Frontiers in Neuroscience. 8:1-11. ISSN: 1662-453X. https://doi.org/10.3389/fnins.2014.00047

MARTZ P, Georgiev P, Wehrend A. 2017. Prolonged second stage labour and consequences of hypoxia in the neonate: A review. Bulgarian Journal of Veterinary Medicine. 22:131-142. ISSN: 1313-3543. https://doi.org/10.15547/bjvm.2012

OROZCO-GREGORIO H, Bonilla-Jaime H, Mota-Rojas D, Trujillo-Ortega ME, RoldanSantiago P, Martínez-Rodríguez R, Borderas-Tordesillas F, Flores-Peinado S, MoraMedina P, Ramírez-Necoechea R. 2012. Effects of subcutaneous administration of caffeine on the physiometabolic profile of low-birthweight neonate piglets. Animal Production Science. 52:981. ISSN: 1836-5787. https://doi.org/10.1071/AN11199 
OROZCO-GREGORIO H, Mota-Rojas D, Alonso-Spilsbury M, Olmos-Hernandez A, Ramirez-Necoechea R, Velazquez-Armenta EY, Nava-Ocampo AA, HernandezGonzalez R, Trujillo-Ortega ME, Villanueva-Garcia D. 2008. Short-term neurophysiologic consequences of intrapartum asphyxia in piglets born by spontaneous parturition. International Journal of Neuroscience. 118:1299-1315. ISSN: 1563-5279. https://doi.org/10.1080/00207450701872846

ROOTWELT V, Reksen O, Farstad W, Framstad T. 2013. Postpartum deaths: Piglet, placental, and umbilical characteristics. Journal of Animal Science. 91:2647-2656. ISSN: 1525-3163. https://doi.org/10.2527/jas.2012-5531

SÁNCHEZ-SALCEDO J, Bonilla-Jaime H, González-Lozano M, Hernández-Arteaga S, Greenwell-Beare V, Vega-Manriquez X, González-Hernández M, Orozco-Gregorio H. 2019a. Therapeutics of neonatal asphyxia in production animals: a review. Veterinárni medicína. 64(05):191-203. ISSN: 1805-9392. https://doi.org/10.17221/86/2018-vetmed

SÁNCHEZ-SALCEDO JA, Orozco-Gregorio H, González-Lozano M, Roldán-Santiago P, González-Hernández M, Ballesteros-Rodea G, Bonilla-Jaime H. 2019b. Caffeine administered to pregnant sows improves piglet vitality, gas exchange and body weight gain. Animal Reproduction Science. 208:1-8. ISSN: 0378-4320. https://doi.org/10.1016/j.anireprosci.2019.106120

SEDDON YM, Cain PJ, Guy JH, Edwards SA. 2013. Development of a spreadsheet based financial model for pig producers considering high welfare farrowing systems. Livestock Science. 157:317-321. ISSN: 1871-1413. https://doi.org/10.1016/j.livsci.2013.07.003

TRUJILLO-ORTEGA ME, Mota-Rojas D, Olmos-Hernández A, González M, Orozco H, Ramírez-Necoechea R, Nava-Ocampo AA. 2007. A study of piglets born by spontaneous parturition under uncontrolled conditions: could this be a naturalistic model for the study of intrapartum asphyxia? Acta Biomedica: Atenei Parmensis. 78(1): 29-35. ISSN: 2531-6745. https://doi.org/10.1080/00207450701872846

VANDERHAEGHE C, Dewulf J, de Kruif A, Maes D. 2013. Non-infectious factors associated with stillbirth in pigs: A review. Animal Reproduction Science. 139:76-88. ISSN: 0378-4320. https://doi.org/10.1016/j.anireprosci.2019.106120

VAN DIJK AJ, Van Rens BTTM, Van der Lende T, Taverne MAM. 2005. Factors affecting duration of the expulsive stage of parturition and piglet birth intervals in sows with uncomplicated, spontaneous farrowings. Theriogenology. 64:1573-1590. ISSN: 0093-691X. https://doi.org/10.1016/j.theriogenology.2005.03.017

YLI BM, Kjellmer I. 2016. Pathophysiology of foetal oxygenation and cell damage during labour. Best Practice \& Research: Clinical Obstetrics \& Gynaecology. 30:9-21. ISSN: 1521-6934. https://doi.org/10.1016/j.bpobgyn.2015.05.004 